hep-th/9908152

IASSNS-HEP-99/77

August, 1999

\title{
A Note On Space Noncommutativity
}

\author{
Zheng Yinf \\ School of Natural Sciences \\ Institute for Advanced Study \\ Olden Lane, Princeton, NJ 08540
}

\begin{abstract}
We consider a two-point spatial lattice approximation to an open string moving in a flat background with $B$ field. It gives a constrained dipole system under the influence of a vector potential. Solving and quantizing this system recover all the essential features of a noncommutative space. In particular, open string interactions induce a canonical product structure on the Hilbert space of the dipole system. It coincides with the usual star product, even though the position operators can be thought of as mutually commuting. Modification of gauge transformations in this noncommutative space also naturally emerges.
\end{abstract}

*email: yin@ias.edu 


\section{Introduction and ideology}

In the passage from classical to quantum mechanics, the physical observables of a system, which are functions on its phase space, become the operators on the Hilbert space of physical states. The canonical commutation relations of Heisenberg

$$
\left[X^{\mu}, P_{\nu}\right]=\imath \hbar \delta_{\nu}^{\mu}
$$

require as well as epitomize this fundamental change. Thus in the quantum world the phase space ceases to be a normal "space," because the notion of it as being made up of well defined "points" becomes invalid (the uncertainty principle). Rather than a function that evaluates at each point of phase space, a physical observable $W(X, P)$ must be understood in its entirety as an operator. This is how algebraic operations on them are performed. In particular, multiplications among operators are associative but generically noncommutative. This is an instance of noncommutative geometry.

By contrast, other parts of the canonical commutation relations

$$
\left[X^{\mu}, X^{\nu}\right]=0
$$

indicate that the coordinates on the configuration space form a set of commuting observable and remain simultaneously measurable. The usual pointbased geometry of the configuration space therefore survives. Wavefunctions, or more generally fields on it, obtain values at each point and algebraic operations are performed pointwise.

It is perhaps only natural to go one step further and pass onto some noncommutative geometry for the configuration space as well. For curiosity's sake it intrigues to see what transpires when wavefunctions and fields become noncommutative objects, but from what sort of physical system would such circumstances arise in the first place? One class of such scenarios have recently been uncovered: the matrix theory [1] naturally accommodates noncommutative tori [2]. Subsequently it is shown how Heisenberg-like commutation relations such as

$$
\left[X^{\mu}, X^{\nu}\right]=\imath \Omega^{\mu \nu}
$$

apply to the coordinate fields on the ends of an open string. In particular, $\Omega$ is nonvanishing if so is the background for the anti-symmetric rank two tensor $B_{\mu \nu}$ [3]. A noncommutative product of functions of the $X$ 's also appear in the operator product expansion on the open string boundary [4, 5]. 
This is of course not the first time noncommutative geometry has been advocated to play a role in (open) string theory. In [6], a noncommutative but associative algebra quite naturally emerged from Type I string theory and was essential in formulating a string field theory. However, this underlying "space" of that algebra is very large and complicated. It is the space of open paths in space-time. Using the terminology for open string quantization, it includes not only the wavefunction of the "center of mass" coordinates, but also those of the infinite tower of oscillators. Somewhere among them one would like to discern the most basic features of the space-time. The aim of this brief note is precisely to extract this piece of information. In section two we truncate the theory of an open string ending on a D-brane to a constrained quantum mechanical model for its two end points? This dipole system inherits a noncommutative product structure on its Hilbert space from the open string interaction. We show that it coincides with the "star" product in deformation quantization in section three. This model allows us also to study how the usual gauge transformations are deformed in noncommutative geometry. Finally, we give some remarks.

\section{The constrained dipole model}

In the conformal gauge, the free action for an open string starting and ending on the same (single) D-brane is

$$
\begin{aligned}
S= & \frac{1}{4 \pi \alpha^{\prime}} \int_{\Sigma} d^{2} \sigma\left[G_{\mu \nu}\left(\dot{Y}^{\mu} \dot{Y}^{\nu}-Y^{\prime \mu} Y^{\prime \nu}\right)+B_{\mu \nu}\left(\dot{Y}^{\mu} Y^{\prime \nu}-Y^{\prime \mu} \dot{Y}^{\nu}\right)\right] \\
& +\int_{\partial_{2} \Sigma} d \tau A_{\mu}(Y) \dot{Y}^{\mu}-\int_{\partial_{1} \Sigma} d \tau A_{\mu}(Y) \dot{Y}^{\mu}
\end{aligned}
$$

Here the subscripts " 2 " and " 1 " on $\partial \Sigma$ label the two boundaries of the open string worldsheet. $G$ and $B$ are constant backgrounds for the metric and rank two anti-symmetric tensor fields respectively. $A$ is the background gauge potential for a constant $U(1)$ field strength on the D-brane. The term involving $B$ is a locally exact, can be replaced by an integral on the boundaries, and absorbed by $A_{\mu} \rightarrow A_{\mu}+B_{\mu \nu} X^{\nu}$. We will do so in the rest of

\footnotetext{
* See also [7].
} 
the paper and henceforth drop any explicit reference to $B$, writing the action more succinctly as

$$
\begin{aligned}
S= & \frac{1}{4 \pi \alpha^{\prime}} \int_{\Sigma} d^{2} \sigma\left(\dot{Y}^{2}-Y^{\prime 2}\right) \\
& +\int_{\partial_{2} \Sigma} d \tau A_{\mu}(Y) \dot{Y}^{\mu}-\int_{\partial_{2} \Sigma} d \tau A_{\mu}(Y) \dot{Y}^{\mu}
\end{aligned}
$$

Unless otherwise specified, we will also restrict our attention to the spatial part of the target space-time for which $F=d A$ is nondegenerate, assuming that the background allows such a decomposition. The full solution of the equation of motion requires the boundary condition

$$
\frac{1}{2 \pi \alpha^{\prime}} G_{\mu \nu} Y^{\prime \nu}=F_{\mu \nu} \dot{Y}^{\nu}
$$

Now we approximate the spatial extent of the open string by a "lattice" of two points, situated at the two boundaries and labeled accordingly. Let the width of the string be $2 / \omega$. Then spatial derivative $Y^{\prime}$ is approximated by $\frac{\omega}{2}\left(Y_{2}-Y_{1}\right)$, and the action (eq. 2.2) by

$$
\begin{aligned}
S= & \int d \tau\left[\frac{1}{4 \pi \omega \alpha^{\prime}}\left(\dot{Y}_{1}^{2}+\dot{Y}_{2}^{2}-\frac{\omega^{2}}{2}\left(Y_{2}-Y_{1}\right)^{2}\right)\right] \\
& +\int d \tau\left(A_{\mu}\left(Y_{2}\right) \dot{Y}_{2}^{\mu}-A_{\mu}\left(Y_{1}\right) \dot{Y}_{1}^{\mu}\right) .
\end{aligned}
$$

With parameters that are more suggestive for particle mechanics,

$$
m=\frac{1}{\pi \omega \alpha^{\prime}}, \quad k=\frac{\omega}{4 \pi \alpha^{\prime}}
$$

the action now reads as ${ }^{\circledast}$

$$
\begin{aligned}
S= & \int d \tau\left[\frac{m}{4}\left(\dot{Y}_{1}^{2}+\dot{Y}_{2}^{2}\right)-\frac{k}{2}\left(Y_{2}-Y_{1}\right)^{2}\right] \\
& +\int d \tau\left(A_{\mu}\left(Y_{2}\right) \dot{Y}_{2}^{\mu}-A_{\mu}\left(Y_{1}\right) \dot{Y}_{1}^{\mu}\right) .
\end{aligned}
$$

It is the action of a dipole of total mass $m$ equally divided among two points of charge \pm 1 linked by a spring with Hooke constant $k$. However, one also

*As usual we use $G$ and $G^{-1}$ to lower, raise, and contract indices. 
has to remember to impose the lattice version of the constraints (eq. 2.3)

$$
\begin{aligned}
C_{1}^{\mu} & \equiv\left(Y_{1}-Y_{2}\right)^{\mu}+\frac{1}{k} G^{\mu \rho} F_{\rho \nu} \dot{Y}_{1}^{\nu} \sim 0 \\
C_{2}^{\mu} & \equiv\left(Y_{2}-Y_{1}\right)^{\mu}-\frac{1}{k} G^{\mu \rho} F_{\rho \nu} \dot{Y}_{2}^{\nu} \sim 0 .
\end{aligned}
$$

There is no secondary constraint. Using

$$
\left\{Y_{2}^{\mu}, \Pi_{\nu}^{2}\right\}=\delta_{\nu}^{\mu}, \quad\left\{Y_{1}^{\mu}, \Pi_{\nu}^{1}\right\}=\delta_{\nu}^{\mu}
$$

and

$$
\begin{aligned}
\Pi_{\nu}^{2} & =\frac{m}{2} G_{\nu \mu} \dot{Y}_{2}^{\mu}+A_{\nu}\left(Y_{2}\right), \\
\Pi_{\nu}^{1} & =\frac{m}{2} G_{\nu \mu} \dot{Y}_{1}^{\mu}-A_{\nu}\left(Y_{1}\right)
\end{aligned}
$$

we find that the Poisson brackets of the $C$ 's are

$$
\begin{aligned}
& \left\{C_{2}^{\mu}, C_{2}^{\nu}\right\}=\frac{4}{m k} F^{\mu \mu^{\prime}}\left(\delta_{\mu^{\prime}}^{\nu}-\frac{1}{m k} F_{\mu^{\prime} \nu^{\prime}} F^{\nu^{\prime} \nu}\right) \equiv T^{\mu \nu}, \\
& \left\{C_{1}^{\mu}, C_{1}^{\nu}\right\}=-T^{\mu \nu}, \\
& \left\{C_{1}^{\mu}, C_{2}^{\nu}\right\}=0 .
\end{aligned}
$$

We also find

$$
\left\{Y_{2}^{\mu}, C_{2}^{\nu}\right\}=\frac{2}{m k} F^{\mu \nu}, \quad\left\{Y_{1}^{\mu}, C_{1}^{\nu}\right\}=-\frac{2}{m k} F^{\mu \nu}
$$

Now following the standard procedure for solving constraint on phase space, we compute the Dirac brackets

$$
\begin{aligned}
\left\{Y_{2}^{\mu}, Y_{2}^{\nu}\right\}_{*} & \equiv\left\{Y_{2}^{\mu}, Y_{2}^{\nu}\right\}-\left\{Y_{2}^{\mu}, C_{2}^{\mu^{\prime}}\right\} T_{\mu^{\prime} \nu^{\prime}}^{-1}\left\{C_{2}^{\nu^{\prime}}, Y_{2}^{\nu}\right\} \\
& =\frac{4}{m^{2} k^{2}} F^{\mu \rho} T_{\rho \rho^{\prime}}^{-1} F^{\rho^{\prime} \nu} \\
& =\left(2 \pi \alpha^{\prime}\right)^{2} F^{\mu \mu^{\prime}}\left(\delta_{\mu^{\prime}}^{\nu}-\left(2 \pi \alpha^{\prime}\right)^{2} F_{\mu^{\prime} \nu^{\prime}} F^{\nu^{\prime} \nu}\right) \\
& \equiv \Omega^{\mu \nu} \\
\left\{Y_{1}^{\mu}, Y_{1}^{\nu}\right\}_{*} & =-\Omega^{\mu \nu}, \\
\left\{Y_{2}^{\mu}, Y_{1}^{\nu}\right\}_{*} & =0 .
\end{aligned}
$$


These are precisely the Dirac brackets for coordinate fields on the string boundaries found in [3].

It turns out to be instructive to use the variables

$$
X^{\mu}=\left(Y_{2}^{\mu}+Y_{1}^{\mu}\right) / 2, \quad S^{\mu}=\left(Y_{2}^{\mu}-Y_{1}^{\mu}\right) / 2 .
$$

In term of them, the quantum commutation relations for the constrained system are

$$
\left[X^{\mu}, S^{\nu}\right]=\imath \frac{1}{2} \Omega^{\mu \nu}
$$

but at the same time

$$
\left[X^{\mu}, X^{\nu}\right]=0=\left[S^{\mu}, S^{\nu}\right]
$$

Therefore the $X$ 's and the $S$ 's separately each make a complete set of commuting observables. Since the $X$ 's are the center of mass coordinates of the dipole system, that they commute seems at first sight contrary to the spirit of noncommutative geometry, Yet we shall show presently that it is precisely in terms of these commuting coordinates that a familiar type of noncommutative algebra naturally emerges.

\section{Space Noncommutativity}

In [6] it is observed that Type I string theory naturally gives rise to a noncommutative product: gluing the right end of one open string to the left end of another yields a new open string. This process is at once obviously noncommutative but associative. It is also much in the spirit of the Chan-Paton factors in resembling the multiplication of matrices through the contraction of adjacent covariant and contravariant indices:

$$
(M N)_{b}^{a} \equiv M_{c}^{a} N_{b}^{c}
$$

Open string fields, however, are big and unwieldy objects, and there are varying schemes to implement the actual product. However, any good product should have one thing in common. It should be compatible with the way open strings interact, just as in field theory pointwise multiplication of fields (wavefunctions) represent their pointwise interaction in a second quantized theory. All purely open string interactions can be reduced to the process of two open strings joining together at one boundary and merge into another 
open string. This is what a good product of open string fields should describe. What happens to the interior of the strings must be complex and one needs to perform gauge fixing to describe it, but at least the wavefunctions of two "adjacent" end points get "contracted." On the other hand, in reducing the open string to the dipole system (eq. 2.6), the end points are what is left of the string. Thus string interaction induces a canonical product on the Hilbert space $\mathcal{H}$ of the dipole system.

Because the $X$ 's form a complete set of commuting observables, states in $\mathcal{H}$ space can be written as functions of them. To write out the sought product, however, it is convenient to choose another basis. In light of (eq. 2.12), $\mathcal{H}$ has a tensor product structure $\mathcal{H}_{2} \otimes \mathcal{H}_{1}$. $\mathcal{H}_{i}$ is the Hilbert space associated with the $\mathrm{i}$-th endpoint, furnishing a representation for $Y_{i}$ in (eq. 2.12). $\mathcal{H}_{2}$ and $\mathcal{H}_{1}$ are canonically isomorphic. Choosing a basis of states $|z\rangle_{i}$ for $\mathcal{H}_{i}$, the product on $\mathcal{H}$ is defined by

$$
\left(\left|z_{2}\right\rangle_{2} \otimes\left|z_{1}\right\rangle_{1}\right) *\left(\left|z_{2}^{\prime}\right\rangle_{2} \otimes\left|z_{1}^{\prime}\right\rangle_{1}\right) \rightarrow\left(\left\langle z_{1}|| z_{2}^{\prime}\right\rangle\right)\left|z_{2}\right\rangle_{2} \otimes\left|z_{1}^{\prime}\right\rangle_{1}
$$

The explicit calculation is carried out by first choosing from the $Y_{2}$ 's a maximal set of commuting operators $\left\{Z_{2}\right\}$. The corresponding operator $\left\{Z_{1}\right\}$ from the $Z_{1}$ 's is similarly a maximal commuting set in light of (eq. 2.12). We also divide the $X$ 's into $\hat{X}=\left(Z_{1}+Z_{2}\right) / 2$ and $\tilde{X}$ being the rest. The eigenstates of the $Z_{i}$ 's furnish a set of basis for $\mathcal{H}_{i}$ suitable for use in (eq. 3.1). Using (eq. 2.12), one find the conversion matrix elements between this and the $x$ basis that diagonalizes the $X$ 's:

$$
\left\langlex \left|\left(\left|z_{2}\right\rangle_{2} \otimes\left|z_{1}\right\rangle_{1}\right)=\delta\left(\hat{x}-\left(z_{2}+z_{1}\right) / 2\right) \exp \left(\frac{\imath}{4} \Omega_{\mu \nu}^{-1} \tilde{x}^{\mu}\left(z_{2}^{\nu}-z_{1}^{\nu}\right)\right) .\right.\right.
$$

Using this, we find that the product in (eq. 3.1) is explicitly given by

$$
\left(\psi_{1} * \psi_{2}\right)[x]=\left.\exp \left(\frac{\imath}{2} \Omega^{\mu \nu} \frac{\partial}{\partial x^{\prime \mu}} \frac{\partial}{\partial x^{\prime \prime \nu}}\right) \psi_{1}\left(x^{\prime}\right) \psi_{2}\left(x^{\prime \prime}\right)\right|^{x^{\prime \prime}=x^{\prime}=x} .
$$

It coincides with the star product studied by mathematicians in deformation quantization.

This product structure also implies two maps from a state $\psi(x)$ in $\mathcal{H}$ to an operator $\psi_{*}$ acting on $\mathcal{H}$. These maps are one-to-one but not onto. To see what kind of operators one can obtain from a wavefunction $\psi(x)$, note 
that by (eq. 2.14) $S$ is realized as an operator on $\mathcal{H}$ as follows:

$$
S=-\frac{\imath}{2} \Omega^{\mu \nu} \frac{\partial}{\partial x^{\nu}}
$$

Therefore we can rewrite (eq. 3.3) as

$$
\left(\psi_{1} * \psi_{2}\right)[x]=\psi_{1}(X+S) \psi_{2}(x) \equiv \psi_{1 *} \psi_{2}(x)
$$

with

$$
\psi_{*} \equiv \psi\left(Y_{2}\right)=\psi(X+S) .
$$

being understood as an operator. It is easy to convince oneself that this map is an algebra homomorphism:

$$
\psi_{1 *} \psi_{2 *}=\left(\psi_{1} * \psi_{2}\right)_{*} .
$$

Treating (eq. 3.3) as an operator $\psi_{*^{\prime}}$ acting on the state $\psi_{1}$ gives us an analogous map

$$
\psi_{*^{\prime}} \equiv \psi\left(Y_{1}\right)=\psi(X-S)
$$

with the property

$$
\psi_{1 *^{\prime}} \psi_{2 *^{\prime}}=\left(\psi_{2} * \psi_{1}\right)_{*^{\prime}} .
$$

In more abstract language, $\psi_{1 *}$ and $\psi_{2 *^{\prime}}$ act on the same module $\mathcal{H}$ from left and right respectively. They always commute.

We can now deduce how gauge transformation would affect the constrained dipole. The action (eq. 2.6) by itself implies that under an infinitesimal transformation $A_{\mu} \rightarrow A_{\mu}+\partial_{\mu} \alpha(x)$, the wavefunction changes its phase by a position dependent $\exp ^{\imath \alpha\left(Y_{2}\right)-\imath \alpha\left(Y_{1}\right)}$. The constraints (eq. 2.7) change the story by making the $Y$ 's noncommuting operators. Under an infinitesimal $\alpha$, the wavefunction changes by

$$
\delta \psi(x)=i\left(\alpha_{*}-\alpha_{*^{\prime}}\right) \psi(x)=i(\alpha(x) * \psi(x)-\psi(x) * \alpha(x)) .
$$

For finite $\alpha$,

$$
\psi(x) \rightarrow \exp ^{* \imath \alpha(x)} * \psi(x) * \exp ^{*(-\imath \alpha(x))}=\exp ^{\imath \alpha_{*}} \exp ^{-\imath \alpha_{*^{\prime}}} \psi(x)
$$

where

$$
\exp ^{* f(x)} \equiv \sum_{n=0}^{\infty} \frac{1}{n !} f(x) * f(x) * \cdots \mathrm{n} \text { times } \cdots * f(x) .
$$

The originally Abelian $U(1)$ gauge group now becomes non-Abelian.

${ }^{*}$ On account that $\Omega$ is anti-symmetric, there is no operator ordering ambiguity in treating $\psi_{1}(X+S), \psi_{2}\left(Y_{2}\right)$, or the like, as formal power series. 


\section{Remarks}

After the work reported here had been completed, a preprint appeared [7] that discussed some related issues and employed a quantum mechanical model resembling the one used here but without the constraints (eq. 2.7).

Subsequently, another preprint on related subjects appeared [5]. Among other issues, it studied gauge bosons on D-branes with constant background $\mathrm{B}$ field in relation to noncommutative geometry. By contrast, gauge boson dynamics is not visible in the model studied in the present paper. This is because in throwing out the bulk degrees of freedom to obtain (eq. 2.6) one removes all the oscillator parts of the string field. Hence only a scalar particle, namely the tachyon, remains in (eq. 2.6). One can try to include the photon with a more elaborate model. On the other hand, while its physics is relatively simple, (eq. 2.6) can serve as a systematic and tractable "probe" for noncommutative "space" in a way akin to how D0-branes can probe various objects in string theory.

\section{Acknowledgment}

We would like to thank P. Aschieri, K. Bardakci, B. Morariu, H. Ooguri, N. Seiberg and E. Witten for useful discussion. The research reported here is supported by NSF grant PHY-9513835. Most of it was done while the author was visiting the particle theory group at Lawrence Berkeley Laboratory. Their hospitality is hereby gratefully acknowledged.

\section{References}

[1] T. Banks, W. Fischler, S.H. Shenker and L. Susskind, "M theory as a matrix model: A Conjecture," Phys. Rev. D55, 5112 (1997) hepth/9610043.

[2] A. Connes, M.R. Douglas and A. Schwarz, "Noncommutative geometry and matrix theory: Compactification on tori," JHEP 02, 003 (1998)

* Aspects of their work had been reported by those authors earlier at the U. Penn. conference in May as well as String 99. 
hep-th/9711162. M.R. Douglas and C. Hull, "D-branes and the noncommutative torus," JHEP 02, 008 (1998) hep-th/9711165.

[3] C. Chu and P. Ho, "Constrained quantization of open string in background B field and noncommutative D-brane," hep-th/9906192.

[4] V. Schomerus, "D-branes and deformation quantization," JHEP 06, 030 (1999) hep-th/9903205.

[5] N. Seiberg and E. Witten, "String Theory and Noncommutative Geometry," hep-th/9908142.

[6] E. Witten, "Noncommutative Geometry And String Field Theory," Nucl. Phys. B268, 253 (1986).

[7] D. Bigatti and L. Susskind, "Magnetic fields, branes and noncommutative geometry," hep-th/9908056. 